\title{
The first childhood case with coexisting Hashimoto thyroiditis, vitiligo and autoimmune hepatitis
}

\author{
Melikşah Keskin ${ }^{1}$, Şenay Savaş-Erdeve ${ }^{1}$, Ferda Özbay-Hoşnut² ${ }^{2}$ Erdal Kurnaz ${ }^{1}$, Semra Çetinkaya ${ }^{1}$, \\ Zehra Aycan ${ }^{1}$ \\ ${ }^{1}$ Clinic of Pediatric Endocrinology and 2Pediatric Gastroenterology Clinic, Dr. Sami Ulus Obstetrics and Gynecology and \\ Pediatrics Training and Research Hospital, Ankara, Turkey. \\ E-mail: meliksah.keskin@hotmail.com \\ Received: 19th October 2015, Revised: 5th April 2016, Accepted: 9th June 2016
}

SUMMARY: Keskin M, Savaş-Erdeve Ş, Özbay-Hoşnut F, Kurnaz E, Çetinkaya $S$, Aycan Z. The first childhood case with coexisting Hashimoto thyroiditis, vitiligo and autoimmune hepatitis. Turk J Pediatr 2016; 58: 432-435.

Hashimoto thyroiditis (HT) is the most common pediatric autoimmune endocrine disorder. It results in autoimmune-mediated thyroid gland destruction and is an organ-specific, typical autoimmune disease. The presence of antithyroid antibodies and the typical pattern on ultrasonography indicate the diagnosis. It is also frequently seen together with other autoimmune disorders including type 1 insulin-dependent diabetes, celiac disease, alopecia and vitiligo.

Autoimmune hepatitis (AIH) is a chronic type of liver injury with an immune etiology that can frequently cause end-stage liver disease if left untreated. Autoimmune hepatitis patients may present with hepatitis, and the laboratory tests in the absence of other etiology usually reveal a positive immune serology together with elevated immunoglobulins and abnormal liver histology. It is interesting that HT and AIH are rarely seen together although both have an autoimmune etiology.

A 14-year-old male who was being followed-up for vitiligo presented with symptoms of a swelling at the neck and fatigue. He was diagnosed with HT after the tests and the liver enzymes were found to be high. The patient was also diagnosed with AIH after tests revealed that the liver enzyme elevation had continued for longer than six months. The thyroid functions and liver enzymes returned to normal and the symptoms decreased after sodium L-thyroxine replacement together with steroid and azathioprine treatment.

We present this case as we believe it is the first pediatric patient diagnosed with HT, AIH and vitiligo.

Key words: Hashimoto thyroiditis, autoimmune hepatitis, vitiligo, autoimmune disorder.

Hashimoto thyroiditis (HT) is the most common pediatric autoimmune endocrine disorder. It results in autoimmune-mediated thyroid gland destruction and is an organ-specific, typical autoimmune disease. The presence of antithyroid antibodies and the typical pattern on ultrasonography indicate the diagnosis. The patient may be suffering from various thyroid problems from transient hyperthyroidism to overt hypothyroidism at presentation ${ }^{1,2}$. The pathology consists of lymphocytic cell infiltration, follicular atrophy and hyperemia in the thyroid together with atrophy of tissue and follicular cell oncocytic metaplasia. The incidence is much higher in patients with other autoimmune disorders such as diabetes mellitus type 1, systemic lupus erythematosus, rheumatoid arthritis and Addison's disease, classified as autoimmune polyendocrine syndromes ${ }^{3}$.

Autoimmune hepatitis $(\mathrm{AIH})$ can be seen at any age and is generally a progressive and chronic hepatitis of unknown etiology. The probable etiology of this multifactorial polygenic disorder is thought to be the interaction between a trigger and various environmental factors in an already genetically susceptible individual. Most patients are female and the clinical presentation can vary from minor symptoms all the way to acute liver failure 4 . 
The coexistence of HT, AIH and vitiligo is quite rare and has previously been reported in only one adult case as far as we are aware ${ }^{5}$. However, this coexistence may not be as rare as it is thought to be.

\section{Case Report}

A 14-year-old male patient who was being followed-up at the dermatology department for vitiligo presented with symptoms of swelling of the neck and fatigue. His history revealed that he had been followed-up for vitiligo since the age of 8 years and did not use any medication. There was no kinship between the parents and no significant family history. The physical examination of the patient at admission revealed a fully cooperative patient in good general condition. Body temperature was $36.5^{\circ} \mathrm{C}$, apical heart peak $86 /$ minute, respiratory rate $16 /$ minute and blood pressure 105/70 mmHg. Bilateral thyroid lobes were palpable and hypopigmented lesions were present on the entire body. There was no organomegaly and other physical examination findings were normal. Laboratory investigations revealed hemoglobin $13.3 \mathrm{~g} / \mathrm{dl}$ (9.5-14.8), WBC $5.600 \times 10^{3} / \mu \mathrm{L}(5.1-15.5)$, platelet 173 $\times 10^{3} / \mu \mathrm{L}$ (159-353), blood urea nitrogen (BUN) $6 \mathrm{mg} / \mathrm{dl}(0-23)$, and creatinine 0.61 $\mathrm{mg} / \mathrm{dl}$ (0.6-1.2). The serum TSH was 6.44 $\mu \mathrm{IU} / \mathrm{ml}$ (0.6-5.5), free-T4 $1.11 \mathrm{ng} / \mathrm{dl}(0.8-$ 1.9), anti-thyroid peroxidase (TPO) $1080 \mathrm{IU} /$ $\mathrm{ml}$ (0-60), and anti-thyroglobulin (anti-TG) $64.7(0-60) \mathrm{IU} / \mathrm{ml}$. Thyroid ultrasonography revealed a thyroid volume of $9.9 \mathrm{~cm}^{3}$ and a heterogeneous appearance secondary to hypoechogenic infiltration in both thyroid lobes. The patient was diagnosed with HT with these findings. The serum aspartate aminotransferase level (AST) was $63 \mathrm{U} / \mathrm{L}$ (0-27), alanine aminotransferase (ALT) 74 $\mathrm{U} / \mathrm{L}(<29)$, alkaline phosphatase (ALP) 106, glutamyltranspeptidase (GGT) 21 IU/L (2064), total bilirubin 0.6, indirect bilirubin 0.3 , total protein $8 \mathrm{gr} / \mathrm{dl}$ (6.4-8.3), and albumin $4.2 \mathrm{~g} / \mathrm{dl}$ (3-4.8). Previous laboratory results of the patients requested for other complaints revealed elevated transaminase levels for over six months. The relevant requested tests revealed that hepatitis B, hepatitis C, cytomegalovirus (CMV) and Ebstein-Barr (EBV) serologies were negative. The serum alpha-1 antitrypsin level was normal and celiac serology (tissue transglutaminase IgA and IgG, anti-endomycium Ig A) was negative. Serum ceruloplasmin level was $29.9 \mathrm{mg} / \mathrm{dl}$ (22-58) and copper level in 24-hour urine $12.7 \mu \mathrm{g} /$ day (3-35). The eye examination was normal. The patients' serum Ig G level was $2310 \mathrm{mg} / \mathrm{dl}$ (854-1750), IgM $87.5 \mathrm{mg} / \mathrm{dl}$ (78.8-370), C3 $1.01 \mathrm{~g} / \mathrm{l}(0.83-1.77), \mathrm{C} 40.172 \mathrm{~g} / 1$ (0.12-0.4), positive for anti-nuclear antibodies (ANA) (1/80 titer) and anti-double-stranded DNA (Anti-ds-DNA) (1/160 titer) and negative for anti-liver kidney microsomal antibodies (LKM1) and anti-smooth muscle antibodies (ASMA).

Minimal hepatomegaly was found on ultrasonographic analysis of the liver and a fine granular appearance was observed in the liver parenchyma. There was portal and periportal hepatitis with plasma cell at the interface, lymphocytic-plasmacytic infiltration and mild fibrosis on histology evaluation. Magnetic resonance cholangiopancreatography (MRCP) was normal. These results (ANA $\geq 1: 80$ was 2 points, Ig $G$ level $>1.10$-fold was 2 points, typical autoimmune hepatitis findings on liver biopsy was 2 points, exclusion of viral hepatitis was 2 points) indicated a total autoimmune hepatitis score of 8 points and the patient was diagnosed with autoimmune hepatitis ${ }^{6}$.

The diagnosis was HT, AIH, and vitiligo. Levothyroxine was started at a dose of 50 $\mathrm{mg} /$ day, prednisolone $2 \mathrm{mg} / \mathrm{kg} /$ day and azathioprine (AZT) $1 \mathrm{mg} / \mathrm{kg}$. The liver enzymes returned to normal levels on the tenth day of treatment. Prednisolone was tapered slowly to a maintenance dose of $5 \mathrm{mg} /$ day whereas AZT was continued at the same dose following remission. The transaminase levels were normal and autoantibodies negative during 3 years of follow-up.

\section{Discussion}

Hakaru Hashimoto described HT in 1912. However, it was in the 1950 s that its relationship with autoimmunity was shown. HT development is not fully understood but requires the combination of an immune defect, genetic susceptibility and environmental factors $^{3}$. The condition frequently coexists with other autoimmune diseases that include type1 insulin-dependent diabetes, coeliac disease, alopecia, and vitiligo ${ }^{2}$. Our case presented with fatigue while being followed-up with a 
diagnosis of vitiligo and was diagnosed with HT and AIH.

Hashimoto thyroiditis (HT) coexistence with $\mathrm{AIH}$ is rare and it was reported only in 2 previous patients as far as we know 5,7 . The first case was a 44-year-old male who was diagnosed with $\mathrm{AIH}$ as a result of high liver function tests performed for fatigue while being followed-up with HT. The laboratory investigations revealed ALT of $977 \mathrm{IU} / \mathrm{L}$ and AST 1840 IU/L. Anti-nuclear antibody was found to be positive after tests for hepatitis $\mathrm{A}, \mathrm{B}, \mathrm{C}, \mathrm{D}, \mathrm{E}, \mathrm{CMV}$, and EBV, some of the conditions that can cause acute hepatitis, were all negative. Minimal hepatomegaly was observed on liver ultrasonography. Liver biopsy showed portal and periportal hepatitis, dilated portal vein and lymphoplasmacytic infiltration. The patient was diagnosed with $\mathrm{AIH}$ with these findings and started prednisolone treatment at a dose of $25 \mathrm{mg} /$ day. ALT and ALT levels were reported to return to normal afterwards.

The second case in the literature is a 52 -year-old male patient who presented with symptoms of fatigue, weakness, itching and jaundice while being followed-up with HT and vitiligo. The laboratory analyses revealed ALT $278 \mathrm{IU} / \mathrm{L}$, AST $228 \mathrm{IU} / \mathrm{L}$, ALP $512 \mathrm{IU} / \mathrm{L}$, GGT $1315 \mathrm{IU} / \mathrm{L}$, Ig G 36.6 g/L (7-16) and Ig M 3.5 g/L (0.42.3). ANA, ASMA, Anti-ds DNA were positive. Liver biopsy results were consistent with $\mathrm{AIH}$ and primary biliary cirrhosis. The patient was started $30 \mathrm{mg} /$ prednisolone, $50 \mathrm{mg}$ azathioprine and $1250 \mathrm{mg}$ ursodeoxycholic acid (UDCA) daily. Following remission, the prednisolone was decreased to $5 \mathrm{mg}$ /day maintenance dose and the azathioprine and UDCA were continued at the same dose.

The estimated prevalence of AIH in the Western population is $10-50 / 100,000$, making it fairly rare. It has a strong predominance for middleaged women ${ }^{8}$. The female:male ratio is $4: 1$ for type $1 \mathrm{AIH}$ and 10:1 for type $2 \mathrm{AIH}^{4}$. However, all 3 cases including our case where HT and AIH coexist are males. The other patients were diagnosed at adulthood but our case was diagnosed at childhood. The presentation signs can be nonspecific and include fatigue, lethargy, nausea, abdominal pain and itching of varying severity ${ }^{4}$. Our case presented with fatigue and was diagnosed with $\mathrm{AIH}$ following the investigations for repeatedly elevated liver function tests. Many patients also experience arthralgia but have no arthritis ${ }^{9}$. This arthralgia mostly involves the small joints ${ }^{4}$. Our patient did not have this symptom. Physical examination may be normal or reveal hepatomegaly, jaundice and other chronic liver disease symptoms in addition to splenomegaly ${ }^{4}$. There were no chronic liver disease signs in our patient, possibly due to the early diagnosis.

The diagnosis of AIH is a based on a series of positive and negative criteria. These include increase in transaminase levels, positive autoantibodies (ANA and/or SMA $\geq 1: 20$ in type $1 \mathrm{AIH}$; anti-LKM1 $\geq 1 / 10$ and positive anti-LC1 in type $2 \mathrm{AIH}$; positive anti-SLA in type 1 and $2 \mathrm{AIH}$ ), increased serum IgG, interphase hepatitis or multilobular collapse on liver biopsy and exclusion of viral causes, exclusion of Wilson's Disease and a normal cholangiogram ${ }^{10}$. There are two main types of the disorder according to the autoantibodies present. Type $1 \mathrm{AIH}$ is the classic form and is seen mostly in young females. It is characterized by the presence of ANA and/or anti-SMA and a good response to immunosuppression ${ }^{11}$. Type $2 \mathrm{AIH}$ is characterized by the presence of liver-anti-LKM-1 and is usually associated with thyroid and gastric parietal cell (GPC) antibodies. It usually starts at childhood with a second peak between the ages of 35 and 65 years. It has an acute clinical onset and progresses quickly to cirrhosis with 40$70 \%$ of the patients cirrhotic at the time of diagnosis. Response to immunosuppression and the prognosis are not as good as Type$1^{11}$. Our case was diagnosed as Type $1 \mathrm{AIH}$. It is known that anti-ds-DNA can be positive in these cases ${ }^{12}$. The clinical and laboratory findings of autoimmune hepatitis and systemic lupus erythematosus cases can be similar, as in our case. Liver biopsy plays a major role in differentiating these two disorders in such cases. The liver biopsy shows characteristic lesions in patients with $\mathrm{AIH}$, as seen in our case, including interface hepatitis, rosetting of hepatocytes, emperipolesis and consecutive to inflammation fibrosis 13 .

Autoimmune hepatitis is known to be associated with primary biliary cirrhosis and primary sclerosing cholangitis. However, there are very few reports on the association of $\mathrm{AIH}$ 
with autoimmune disorders other than these two conditions ${ }^{4}$. The MRCP of our case was normal. There is only one case in the literature where the coexistence of AIH, primary biliary cirrhosis and vitiligo has been reported. Thyroid disorders (Hashimoto and Graves disease), celiac disease, MS and other autoimmune conditions have been reported to be associated with $\mathrm{AIH}^{4}$. Our case was diagnosed with $\mathrm{AIH}$ coexisting with HT.

We believe our case is the first to be reported with the diagnosis of HT and coexisting AIH in childhood. Our data suggest that a possible association with $\mathrm{AIH}$ should be considered in patients affected by HT with repeated high aminotransferase levels.

\section{REFERENCES}

1. Lee HS, Hwang JS. The natural course of Hashimoto's thyroiditis in children and adolescents. J Pediatr Endocinol Metab 2014; 27: 807-812.

2. Radetti G. Clinical aspects of Hashimoto's thyroiditis. Endocr Dev 2014; 26: 158-170.

3. Pyzik A, Grywalska E,Matyjaszek-Matuszek B, Roliński J. Immune disorders in Hashimoto's thyroiditis: What do we know so far?. J Immunol Res 2015; 2015: 979167.

4. Nadhem ON, Al Janabi M, Omer AR, Wan B. Autoimmune hepatitis with multiple sclerosis and graves disease: coincidence or association?. Case Rep Gastroenterol 2014; 8: 319-323.
5. Aksoy EK, Yılmaz B, Köklü S. Autoimmune hepatitis/ primary biliary cirrhosis overlap syndrome developed in a patient with vitiligo and hashimoto thyroiditis. Eur J Gastroenterol Hepatol 2013; 25: 121-122.

6. Hiejima E, Komatsu H, Sogo T, Inui A, Fujisawa T. Utility of simplified criteria for the diagnosis of autoimmune hepatitis in children. J Pediatr Gastroenterol Nutr 2011; 52: 470-473.

7. Amenduni T, Bellitti P, Carbone A, et al. Unusual association of Hashimoto's thyroiditis with autoimmune hepatitis. Thyroid 2007; 17: 1307-1308.

8. Manns MP. Autoimmune hepatitis: the dilemma of rare diseases. Gastroenterology 2011; 140: 1874-1876.

9. Lohse AW, Mieli-Vergani G. Autoimmune hepatitis. J Hepatol 2011; 55: 171-182.

10. Mieli-Vergani G, Heller S, Jara P, et al. Autoimmune hepatitis. J Pediatr Gastroenterol Nutr 2009; 49: 158164.

11. Mieli-Vergani G and Vergani D. In: Kelly D, ed. Diseases of the Liver and Biliary System in Children, 3rd edition. New Jersey: Blackwell Publishing, 2008: 191-207.

12. Czaja AJ, Morshed SA, Parveen S, Nishioka M. Antibodies to single-stranded and double-stranded DNA in antinuclear antibody-positive type 1-autoimmune hepatitis. Hepatology 1997; 26: 567-572.

13. Beisel C, Weiler-Normann C, Teufel A, Lohse AW. Association of autoimmune hepatitis and systemic lupus erythematodes: a case series and review of the literature. World J Gastroenterol 2014; 20: 1266212667. 